\title{
Magnetized Bianchi type III massive string cosmological models in general relativity.
}

\begin{abstract}
The present study deals with Bianchi type III string cosmological models with magnetic field. The magnetic field is assumed to be along $\mathrm{z}$ direction. Therefore $\mathrm{F} 12$ is only the nonvanishing component of electromagnetic field tensor $F \mathrm{ij}$. The expansion $(\theta)$ in the model is assumed to be proportional to the shear $(\sigma)$. To get determinate solution in term of cosmic time, we have solved the fields equations in two cases (i) Reddy and (ii) Nambu string. The physical and geometrical behaviour of these models is discussed.
\end{abstract}

Keyword: LRS Bianchi type III models; Magnetic field; Massive string. 